\title{
THE CONSPICUITY BENEFITS OF BICYCLE TAILLIGHTS IN DAYLIGHT
}

\author{
Darlene E. Edewaard, Ellen C. Szubski, Richard A. Tyrrell, \\ Andrew T. Duchowski \\ Clemson University, Clemson, SC, USA \\ Email: dedewaa@clemson.edu
}

\begin{abstract}
Summary: Bicyclists risk being injured or killed in crashes with motor vehicles, even during the daytime. Therefore, cyclists must help approaching drivers detect and recognize their presence. The present study examined the conspicuity benefits of bicycle taillights during the daytime. Participants' eye movements were recorded as they searched for vulnerable road users in videos recorded from a driver's perspective in a moving vehicle. Five of the videos contained a bicyclist who displayed one of five taillight configurations. The distance from which each participant first glanced at the bicyclist was recorded, as was the distance from which the participant pressed a button to indicate that a bicyclist was present. The results indicated that the participants first fixated on the bicyclist at a distance that was 2.7 times greater than the distance at which they responded to recognizing the bicyclist. Additionally, the bicyclist was recognized from significantly greater distances when using a flashing or steady seat post-mounted taillight than when no taillight was displayed. These findings confirm earlier research that bicycle taillights can enhance drivers' ability to recognize bicyclists during daylight.
\end{abstract}

\section{INTRODUCTION \& OBJECTIVES}

In 2016, 840 bicyclist fatalities were reported in the United States, and approximately half of these fatalities resulted from crashes that occurred during daylight hours (NHTSA, 2018). Through investigating bicyclist crash reports, Hutchinson and Lindsay (2009) indicated that collisions in which bicyclists are struck from behind by approaching vehicles are the most frequent type of bicyclist/motor vehicle crashes. Too often, drivers do not recognize that a bicyclist is present in the roadway in time to avoid a collision. Thus, a key question concerns what steps bicyclists could take to enhance their conspicuity to drivers approaching from behind.

A growing body of literature indicates that bicycle taillights provide conspicuity benefits for bicyclists while riding at night (Edewaard et al., 2017; Koo \& Dunne, 2012; Koo \& Huang, 2015; Watts, 1984). In terms of daytime conspicuity, Edewaard et al. (2019) conducted a closed road study in which participants rated the extent that each bicyclist among an array of four bicyclists was recognizable as a bicyclist. The results indicated that the bicyclists who displayed a functioning taillight were rated as being significantly more recognizable as bicyclists than the bicyclist who displayed a taillight that was turned off. Participants also rated bicyclists who displayed flashing taillights as being more recognizable than a bicyclist who displayed a steady taillight (Edewaard et al., 2019). Wood et al. (2012) did not find a significant difference between participant drivers' recognition distances of bicyclists with flashing or steady headlights at night. However, Edewaard et al. (2017) conducted a similar nighttime open-road study which indicated that the recognition distances to a test bicyclist were over two times greater when a flashing taillight was used than when a steady taillight was present. While flashing taillights appear to offer conspicuity advantages over steady taillights at night, one question that remains 
unanswered is whether steady and flashing taillights can increase the distances from which approaching drivers recognize that a bicyclist is present during the daytime.

Both Edewaard et al. (2016) and Edewaard et al. (2017) found that lights can enhance bicyclist conspicuity when they highlight the rider's pedaling movements. Humans are perceptually sensitive to recognizing the movement patterns of other human beings. This is a perceptual phenomenon called biological motion (or "biomotion") (Johansson, 1973), which has been verified by numerous pedestrian and bicyclist conspicuity studies (for reviews see Kwan \& Mappstone, 2004; Tyrrell et al., 2016). An open question is whether lights mounted to a rider's heels can enhance rider conspicuity in open-road settings during the daytime. Further, the effects of combining a seat post taillight with heel-mounted lights have also not been assessed.

In terms of conspicuity, there is an important difference between detection and recognition. Detection implies that an object is seen, but the observer may not be able to identify the object. Recognition, on the other hand, implies that the observer can identify that object. It is important to understand the differences between detection and recognition distances in order to understand ways in which bicyclists can be more quickly and easily identifiable to drivers and so drivers can predict the bicyclist's path. One way to assess drivers' abilities to detect and recognize bicyclists is by utilizing eye tracking technology, which was used successfully to study the conspicuity benefits of retroreflective material for pedestrians and bicyclists at night (Wood, Tyrrell, Lacherez, \& Black, 2017; Stapleton \& Koo, 2017). To our knowledge, eye trackers have not yet been used to assess the detection and recognition benefits of bicycle taillights during daytime.

The purpose of this study was to assess the daytime conspicuity benefits of bicycle taillights. Participants' eye movements were tracked as they viewed 25 brief videos of roadway scenarios taken from the perspective of a driver, and participants pressed a button when they became confident that a bicyclist was present. Thus, this study quantified both detection and recognition distances to bicyclists during the daytime.

\section{METHODS}

\section{Participants}

Ninety undergraduate students participated in this study. Each was required to have a 20/40 or better binocular visual acuity (Bailey-Lovie) and a log contrast sensitivity of 1.65 or better (PelliRobson) while wearing their presenting optical correction, if any. Eye tracking data were not available from 55 participants due to a variety of causes (e.g., participants failing to follow instructions, eye tracker signal loss). Thus, complete data are available from a total of 35 participants (mean age $=20$ ). Each participant received credit toward their psychology class. All procedures were approved by the Institutional Review Board, and all participants provided informed consent prior to testing.

\section{Design}

This study used a within-subjects design, such that each participant viewed all 25 video clips of a vehicle's forward view of the same roadway environment. Only data from five video clips (the "data videos") were analyzed, and the participants remained naïve to which of the 25 videos were the data videos. The distance that separated the camera vehicle from the test bicyclist at the moment participants first glanced at the test bicyclist (made their first gaze in the Area of Interest 
surrounding the bicyclist) ("first glance distance") and pressed a button indicating that they were confident that they saw a bicyclist ("recognition distance") were measured.

Video Clips. Twenty-five videos were recorded at 1920x1080 resolution with ISO set to 6400 with a Canon EOS 60D DSLR camera that was mounted to the middle of the dashboard inside a 2008 Hyundai Elantra on the same clear, sunny day (at least one hour after sunrise and one hour before sunset). Five of the 25 videos (the "data videos") featured a test bicyclist who wore all black clothing (including a helmet, jersey, leggings, socks, and shoes), and displayed one of five bicycle taillight configurations (see Table 1). Taillights were customized Bontrager Flare RTs, which featured lenses that projected the light rearward (see Figure 1). The test bicyclist cycled on a gray Trek Emonda road bike while keeping both hands on the handlebars and avoiding making extraneous (non-cycling) movements. The road was a 4-lane highway with opposing lanes separated by a median and with a bicycle lane to the right of each outside lane. The camera vehicle approached the test bicyclist from the rear in the right lane and maintained the speed limit of $40 \mathrm{mph}(64.4 \mathrm{~km} / \mathrm{hr})$ along a straight and level stretch of the highway with a maximum sight distance of $620 \mathrm{~m}$. The test bicyclist traveled in the bicycle lane at a constant speed of 12 mph with a cadence of approximately $80 \mathrm{rpm}$. No extraneous bicycles were present during any of the data videos. The data videos were recorded in such a way that the camera vehicle passed the bicyclist near the end of the stretch of roadway (Figure 2). The remaining 20 videos served as distractors containing extraneous bicyclists or pedestrians to prevent the participants from knowing in advance where to look to see a bicyclist. The videos were edited to begin at the same position and played at $60 \mathrm{fps}$. The length of the videos varied between 45 and 50 seconds.

Taillight

Configuration

Control A single taillight - powered off - mounted to the seat post of the bicycle.

Flashing A single taillight operating on an irregular flash pattern mode (see Figure 1) was mounted to the Seat Post seat post of the bicycle.

Steady A single taillight operating on a 25 lumen steady (always-on) mode was mounted to the seat Seat Post post of the bicycle.

Heels Two taillights operating on the 25 lumen steady mode were mounted to the heels of the cyclist's shoes, facing the traffic approaching from behind.

Three taillights total: A single taillight operating in the 25 lumen steady (always-on) mode was

Warping

Triangle

\section{Table 1. The Five Taillight Configurations}
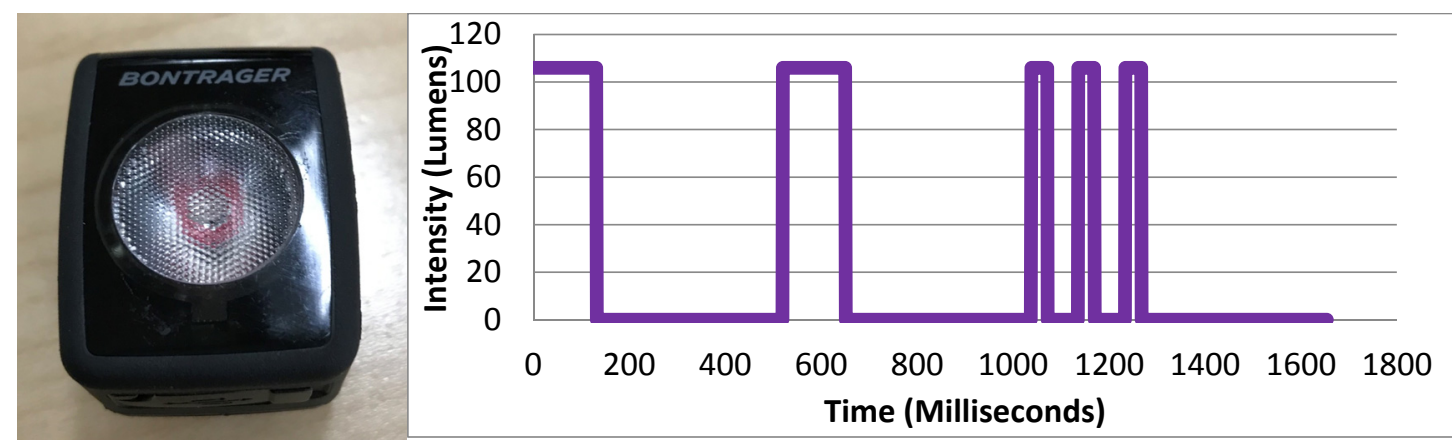

Figure 1. The Bontrager Flare RT taillight, and one cycle of the flash mode used in the Flashing Seat Post configuration. 
Stimulus presentation and eye tracking. The 25 videos were presented on an iMac desktop computer with a 27" Retina 5K display (5120 x 2880 resolution) using the PsychoPy experimental control software package (v1.85.4). An adjustable forehead and chin rest was mounted in front of the test computer to limit participants' head movements (see Figure 2), and participants' eyes were $48 \mathrm{~cm}$ from the monitor to ensure that the visual angles of objects in the video matched the visual angles observed from the camera vehicle. The eye tracker was a monitor-mounted Gazepoint (GP3) Eye Tracker with a binocular camera system, a sampling rate of $60 \mathrm{~Hz}$, and an accuracy of 1 degree.

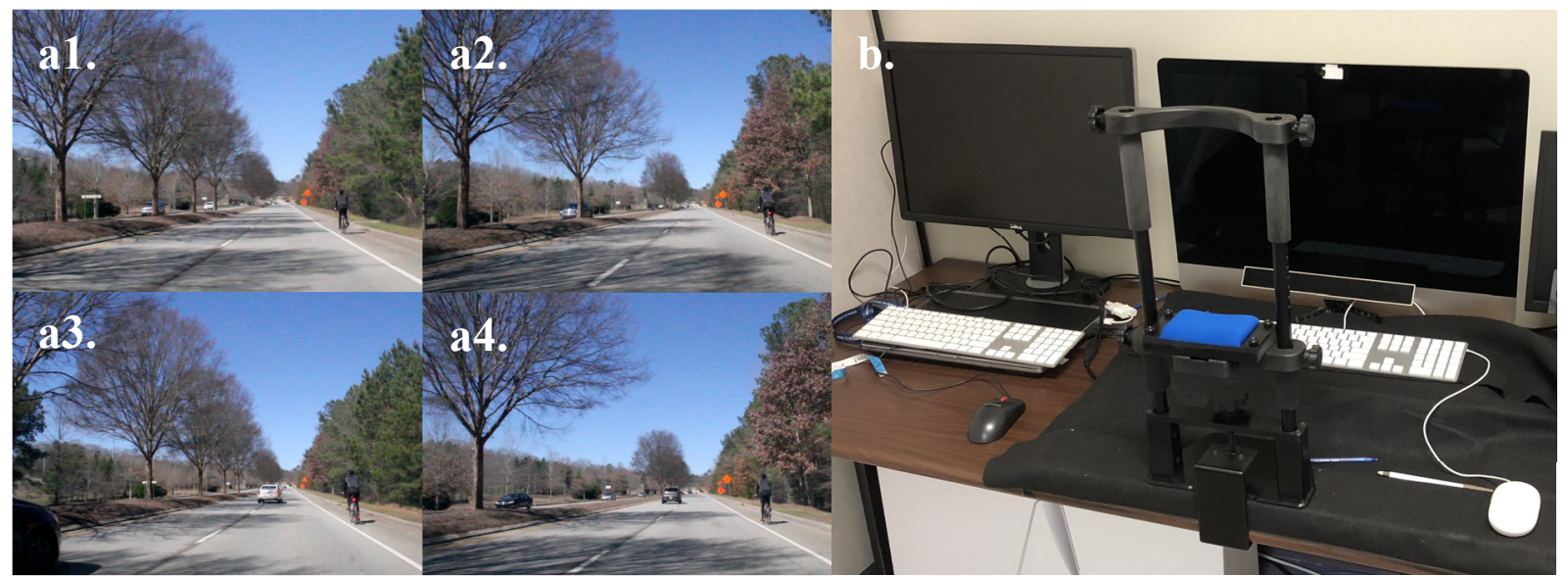

Figure 2. a. Screen shots of Control (a1), Steady Seat Post (a2), Heels (a3), and Warping Triangle (a4). b. The test apparatus.

\section{Procedure}

The participants were tested one at a time. After being positioned in the chinrest, each participant went through a five-point calibration procedure. Once the eye tracker was calibrated, the participants were instructed to look at a fixation cross in the middle of a gray screen for five seconds until a video appeared and began playing. The participants were told to "search for any person(s) (e.g., bicyclists, runners, or walkers) in or near the roadway, not in a motor vehicle." Participants were also told that not every video would contain a person. They were instructed to press the spacebar when they were confident that they saw a person in the videos and could recognize what the person was doing (e.g., biking, running, or walking). After pressing the spacebar, the video was paused, and the participants pressed a button to indicate the type of person(s) that they saw in the videos by pressing "b" for bicyclist(s), " $r$ " for runner(s), "w" for walker(s), or " $n$ " if they did not see any person(s). Participants repeated this process for all 25 videos, which were presented in a randomized order across the participants, and when they were finished responding to all of the videos, they were debriefed, thanked, and dismissed. Each experimental session lasted approximately 30 minutes.

\section{RESULTS}

The first glance data were coded using dynamic areas of interest (dynamic AOIs) that surrounded the test bicyclist in the five data videos. The response times from the first glance and button press data were converted to response distances, which were analyzed using a 2 (First Glance versus Recognition) X 5 (Taillight Configuration: Control, Steady Seat Post, Flashing Seat Post, Heels, and Warping Triangle) repeated measures analysis of variance (ANOVA). 
The main effect of First Glance versus Recognition was significant, $F(1,34)=379.38, \eta^{2}=.918$, $p<.001$. Averaged across the five taillight configurations, participants first glanced at the test bicyclist from significantly greater distances $(M=470.8 \mathrm{~m} ; S D=74.3 \mathrm{~m})$ than those at which they pressed the spacebar in response to recognizing the bicyclist $(M=177.4 \mathrm{~m} ; S D=108.2 \mathrm{~m})$. The main effect of Taillight Configuration was also significant, $F(4,136)=7.50, \eta^{2}=.181$, $p<.001$. A Bonferroni post-hoc comparison indicated that when averaging the First Glance and Recognition distances the participants responded to the bicyclist from significantly farther distances when displaying the Flashing Seat Post Taillight $(M=352.6 \mathrm{~m} ; S D=153.4 \mathrm{~m})$ than when displaying the Heels $(M=317.5 \mathrm{~m} ; S D=183.7 \mathrm{~m})$, Warping Triangle $(M=308.5 \mathrm{~m}, S D=$ $180.9 \mathrm{~m})$, and Control $(M=305.2 \mathrm{~m} ; S D=186.9 \mathrm{~m})$ configurations $(p<.05)$. Further, when the Steady Seat Post Taillight $(M=336.6 \mathrm{~m}, S D=161.3 \mathrm{~m})$ was displayed, participants responded to the bicyclist from significantly greater distances than those for the Control configuration $(p<.05)$.

The ANOVA also revealed a significant interaction between Taillight Configuration and First Glance versus Recognition, $F(4,136)=4.38, \eta^{2}=.114, p<.01$ (see Figure 3). Thus, the effect of taillight configuration varied significantly between the first glance data and the recognition data. A one-way repeated measures ANOVA revealed a significant simple effect of Taillight Configuration on the Recognition distances, $F(4,136)=8.66, \eta^{2}=.203, p<.01$. Bonferroni posthoc comparisons revealed that participants recognized the bicyclist from a significantly farther distance when the Flashing Seat Post $(M=226.2 \mathrm{~m}, S D=101.7 \mathrm{~m})$ and the Steady Seat Post $(M$ $=204.3 \mathrm{~m}, S D=102.9 \mathrm{~m})$ configurations were displayed than when the Warping Triangle $(M=$ $153.0 \mathrm{~m}, S D=99.3 \mathrm{~m})$, and Control $(M=140.2 \mathrm{~m}, S D=98.4 \mathrm{~m})$ configurations were displayed $(p<.05)$. In addition, the bicyclist was recognized from a significantly farther distance when displaying the Flashing Seat Post configuration than when the Heels $(M=163.4 \mathrm{~m}, S D=118.3$ $\mathrm{m})$ configuration was displayed $(p<.05)$.

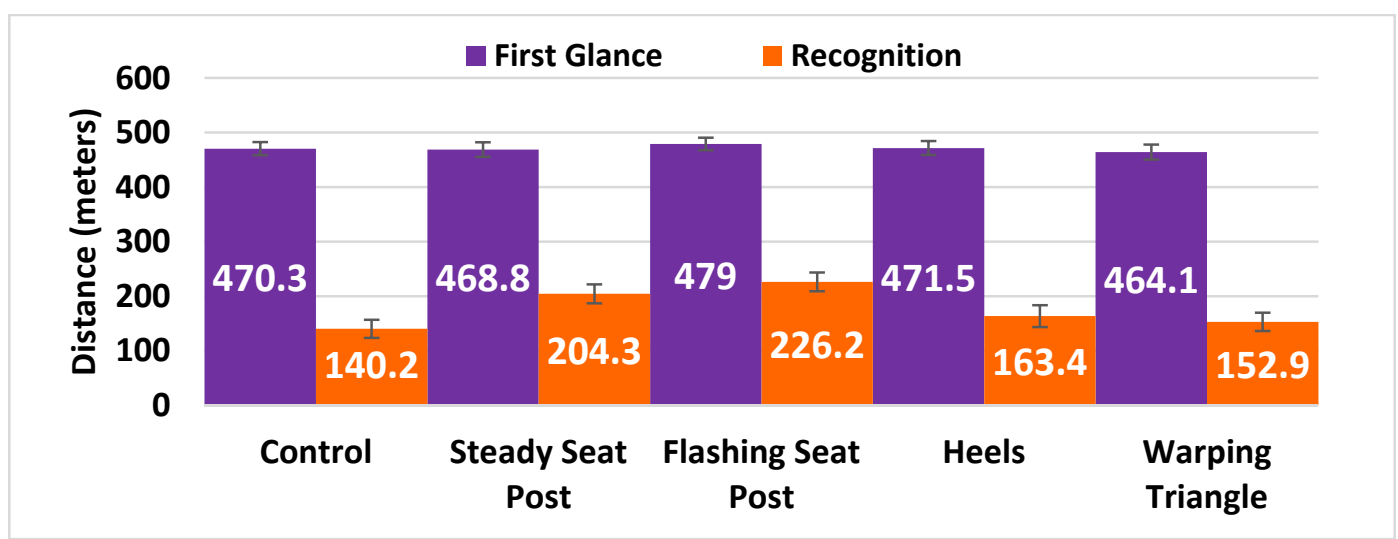

Figure 3. Mean first glance and recognition (button press) distances for each taillight configuration. Error bars represent \pm 1 standard error of the mean.

\section{DISCUSSION}

In this study, participants watched 25 videos of daylight drives along the same roadway. Five of the videos featured the camera vehicle approaching and passing a test bicyclist who displayed one of five taillight configurations. We measured the distances from which participants first glanced at and recognized (via a button press) the bicyclist in these five videos. 
The results of the data obtained from the fixation patterns indicated that participants first glanced at the test bicyclist displaying the five taillight configurations from distances that were significantly greater than those at which they recognized the bicyclist but not significantly different from each other. One reason for this finding may be that in daylight participants could detect the shape of the entire bicyclist from far distances even without the taillight.

Regarding the recognition (button-press) distances, the bicyclist was recognized from significantly farther distances when displaying the flashing and steady seat post configurations than when displaying the Warping Triangle and the Control configurations. The Warping Triangle and Control configurations may have required more processing time due to participants' uncertainty of whether they were looking at a bicyclist, a walker, or a runner before they could recognize the bicyclist. Flashing or steady seat post lights may have facilitated faster recognition by being associated with bicyclists. Participants also recognized the bicyclist from significantly farther distances when a flashing seat post light was used than when a steady light was mounted to each heel of the rider's shoes. This finding contradicts the results of the on-road nighttime study with similar taillight conditions conducted by Edewaard et al. (2017). This may be due to a combination of the videos having only moderate fidelity, and the lenses on the heel lights being aimed away from the approaching camera vehicle during a significant portion of the bicyclist's pedaling (due to cyclic heel motions while pedaling). So, while we believe the present results are ambiguous with regard to the conspicuity benefits of the heel lights, this study confirms that seat post-mounted taillights can enhance bicyclist conspicuity during the daytime, which aligns well with the findings from the daytime taillight study conducted by Edewaard et al. (2019).

While the results of the present study revealed that seat post-mounted taillights can provide conspicuity benefits for bicyclists during the daytime, more research is needed to further explore these benefits. For instance, future studies could be conducted to better understand the optimal light intensity for heel-mounted lights intended for daytime riding environments. Another question that remains unanswered is whether the results of this study would be similar if the data were collected using an in-vehicle (open-road) method. Such on-road studies are required to assess the conspicuity benefits of bicycle taillights in real-world settings, in that participants would encounter the taillight configurations from a moving vehicle in real-time without the visual limitations (e.g., diminished resolution and image instability) that videos present.

\section{CONCLUSION}

The present study explored the detection and recognition benefits of bicycle taillights during the daytime and revealed that flashing or steady seat post-mounted taillights enhance the distances from which a bicyclist is recognized by observers during the daytime. Findings from this study can be used by designers of taillights to better understand how the optical qualities of taillights can affect drivers' abilities to detect and recognize bicyclists who use taillights during the daytime. In addition, the results of this study can be used to educate the bicyclist community on effective ways to use a taillight to enhance their conspicuity to drivers while riding during the daytime. Further research is needed to continue forward progress on these important issues. 


\section{ACKNOWLEDGMENTS}

This research was funded by a contract from Trek Bicycle Corporation. The contributions from several Trek employees, including Kurt Heggland, Scott Kasin, Andy Pliszka, and Jonathan Quenzer, were greatly appreciated. This research would not have been possible without the skilled, diligent, and tireless efforts of Dr. Drea Fekety, as well as our team of Research Assistants, including: Kyle Hitchman, Drayton Tsirkas, and Sarah Wokaty.

\section{REFERENCES}

Edewaard, D.E., Fekety, D.K., Szubski, E.C., \& Tyrrell, R.A. (2019). The rear conspicuity benefits of bicycle taillights and fluorescent clothing for bicyclists riding in daylight. Manuscript in preparation.

Edewaard, D. E., Fekety, D. K., Szubski, E. C., Tyrrell, R. A., \& Rosopa, P. J. (2017). The Conspicuity Benefits of Dynamic and Static Bicycle Taillights at Night. In Proceedings of the Human Factors and Ergonomics Society Annual Meeting (Vol. 61, No. 1, pp. 15671568). Sage CA: Los Angeles, CA: SAGE Publications.

Hutchinson, T.P. \& Lindsay, V.L. (2009). Pedestrian and cyclist crashes in the Adelaide Metropolitan Area (Report \#CASR055). Adelaide, Australia: Centre for Automotive Safety Research.

Johansson, G. (1973). Visual perception of biological motion and a model for its analysis. Attention, Perception, \& Psychophysics, 14(2), 201-211.

Koo, H., \& Dunne, L. (2012). Enhancing visibility in bicycling apparel using integrated flashing LEDs. In Smart Design: First International Conference Proceedings, 41-47. Springer London.

Koo, H.S. \& Huang, X. (2015). Visibility aid cycling clothing: flashing light-emitting diode (FLED) configurations. International Journal of Clothing Science and Technology, 27, 460471.

Kwan, I., \& Mapstone, J. (2004). Visibility aids for pedestrians and cyclists: a systematic review of randomized controlled trials. Accident Analysis \& Prevention, 36(3), 305-312.

National Center for Statistics and Analysis. (2018, May). Bicyclists and other cyclists: 2016 data. (Traffic Safety Facts. Report No. DOT HS 812 507). Washington, DC: National Highway Traffic Safety Administration.

Stapleton, T., \& Koo, H. S. (2017). Bicyclist biomotion visibility aids: a 3D eye-tracking analysis. International journal of clothing science and technology, 29(2), 262-269.

Tyrrell, R. A., Wood, J. M., Owens, D. A., Whetsel Borzendowski, S., \& Stafford Sewall, A. (2016). The conspicuity of pedestrians at night: a review. Clinical and experimental optometry, 99(5), 425-434.

Watts, G.R. (1984). Pedal cycle lamps and reflectors - Some visibility tests and surveys. TRRL Report 1108.

Wood, J.M., Tyrrell, R.A., Marszalek, R., Lacherez, P., Carberry, T., \& Chu, B.S. (2012). Using reflective clothing to enhance the conspicuity of bicyclists at night. Accident Analysis and Prevention, 45, 726-730.

Wood, J. M., Tyrrell, R. A., Lacherez, P., \& Black, A. A. (2017). Night-time pedestrian conspicuity: effects of clothing on drivers' eye movements. Ophthalmic and physiological optics, 37(2), 184-190. 